\title{
Iterative structural identification framework for evaluation of existing structures
}

\author{
Romain Pasquier ${ }^{\mathrm{a}, *}$, Ian F. C. Smith ${ }^{\mathrm{a}}$ \\ ${ }^{a}$ Applied Computing and Mechanics Laboratory (IMAC), School of Architecture, Civil and Environmental Engineering \\ (ENAC), Swiss Federal Institute of Technology (EPFL), CH-1015 Lausanne, Switzerland
}

\begin{abstract}
Evaluation of aging infrastructure has been a world-wide concern for decades due to its economic, ecological and societal importance. Existing structures usually have large amounts of unknown reserve capacity that may be evaluated though structural identification in order to avoid unnecessary expenses related to the repair, retrofit and replacement. However, current structural identification techniques that take advantage of measurement data to infer unknown properties of physics-based models fail to provide robust strategies to accommodate systematic errors that are induced by model simplifications and omissions. In addition, behavior diagnosis is an ill-defined task that requires iterative acquisition of knowledge necessary for exploring possible model classes of behaviors. This aspect is also lacking in current structural identification frameworks. This paper proposes a new iterative framework for structural identification of complex aging structures based on model falsification and knowledge-based reasoning. This approach is suitable for ill-defined tasks such as structural identification where information is obtained gradually through data interpretation and insitu inspection. The study of a full-scale existing bridge in Wayne, New Jersey (USA) confirms that this framework is able to support structural identification through combining engineering judgment with on-site measurements and is robust with respect to effects of systematic uncertainties. In addition, it is shown that the iterative structural-identification framework is able to explore the compatibility of several model classes by model-class falsification, thereby helping to provide robust diagnosis and prognosis.
\end{abstract}

Keywords: Systematic errors, model falsification, knowledge-based reasoning, model-class exploration, behavior diagnosis, prognosis

\section{Introduction}

Due to conservative strategies that are fueled by high risks associated with the construction of large civil structures, most structures today have significant amounts of unknown reserve capacity. In the context of

*Corresponding author. Address: EPFL ENAC IIC IMAC, Station 18, CH-1015 Lausanne, Switzerland. Phone number: +41216932498

Email address: rpasquie@gmail.com (Romain Pasquier)

Preprint submitted to Engineering Structures

September 9, 2015

(C) 2015. This manuscript version is made available under the Elsevier user license

http://www.elsevier.com/open-access/userlicense/1.0/ 
structural health management of existing aging structures, structural identification is attractive for decisionmaking support. The goal of model-based data interpretation is to increase the knowledge of real behavior of complex structures using information provided by behavior measurements. In order to interpret measurement data, physics-based models are used to connect hypotheses of structural behavior to observed behavior and to identify uncertain parameter values of physical properties. This interpretation serves to improve behavior diagnosis and reduce uncertainties associated with behavior prognoses, such as remaining-fatiguelife evaluation. However, diagnosis is an ill-defined task that is performed under conditions of high modeling and measurement uncertainty. In addition, modeling errors are usually systematic, also called epistemic errors as opposed to random errors, thereby increasing interpretation difficulty.

Single-model-updating approaches such as residual minimization have already shown to be inaccurate in the presence of systematic errors since a single optimal model is intrinsically imperfect due to parameter-value compensation [1-4]. Instead, there are always multiple models that are able to explain observations of the behavior of complex structures. Approaches such as probabilistic Bayesian inference accounts for multiple solutions through updating posterior probabilities of parameter values, thereby estimating the uncertainty associated with the parameter values. However, a common assumption in these approaches is that modeling and measurement errors are adequately described by a joint independent zero-mean Gaussian probability density function (PDF) [5-7], which is incompatible with the systematic nature of several modeling uncertainty sources. In addition, some applications incorporate the variance of the joint PDF as a parameter in the identification process [8-10] and others assign an arbitrary value to the variance [11-13]. However, in complex civil structures, modeling uncertainties are often biased and correlated spatially. In addition, defining a statistical model of errors that is not compatible with the true errors leads to biased diagnostics and prognosis $[3,14]$. While Bayesian inference may provide useful support when statistical models of errors are known, it is not robust when aspects such as correlations cannot be quantified.

For the purposes of this paper a model class is defined as a parameterized physics-based model, where parameters are variables whose values need to be identified. Models are instances of model classes. In the context of Bayesian inference, proposals exist to select an optimal model class among a set of possible model classes that gives the best trade-off between data fitting and model-class complexity in order to solve diagnosis and prognosis tasks [9, 15-17]. Some approaches link to Ockham's razor [17, 18], also called principle of parsimony, which asserts that simpler models that are compatible with measurements are preferred over complicated ones. However, simpler models may imply over-idealization of reality and consequently modeling uncertainties. Despite undeniable benefits of this principle to simplify modeling and data-interpretation tasks, the question of the presence of systematic errors in the model class has not been treated explicitly. Although several authors in various fields have pointed out the importance of providing an adequate description of modeling uncertainties associated with the model class [4, 19-22], proposals for robust alternatives to existing approaches are lacking. 
Goulet and Smith [3] proposed an approach that is robust when knowledge of the joint PDF of modeling and measurement errors is incomplete. This approach, named error-domain model falsification (EDMF), combines PDFs of each source of modeling and measurement error and determines conservative probabilistic thresholds that are used to falsify inadequate models. Modeling errors are estimated using engineering heuristics and field observations. They have shown that this approach leads to robust parameter identification in the presence of systematic errors without precise knowledge of the dependencies between modeling errors. Goulet and Smith [3] also demonstrated that the assumption of independence in the common definition of uncertainties in Bayesian inference may bias the posterior distribution of parameter values in the presence of systematic errors. This last observation has also been noted by Simoen et al. [23]. Although Goulet and Smith [3] have observed that EDMF can reveal situations when initial assumptions related to the model class are erroneous by falsifying all model instances, taking advantage of this characteristic for exploring possible model classes of complex structures has not been studied.

Choi and Beven [24] have also observed that model falsification could serve to point out model deficiencies in the search for a better model class. This observation resulted in the proposal of the generalized likelihood uncertainty estimation (GLUE) framework [25] in the field of environmental modeling, which is also affected by large modeling uncertainties. Other examples of model-falsification procedures can be found in this field. Beck [26] presented a framework for analysis of uncertainty and model selection based on recursive search and model discrimination. An approach, called Monte Carlo filtering, is used for discarding sets of inadequate model instances. Also, in the field of geology, Cherpeau et al. [27] proposed a fault-scenario falsification approach using a misfit threshold. However in such examples, systematic errors were not included explicitly.

In the field of civil engineering, structural identification processes are often based on residual minimization approaches [28-31], which may lead to biased results in the presence of unexpected systematic modeling errors. Moon and Aktan [32] proposed a structural identification framework composed of six steps for diagnosis and prognosis of complex structures. The process starts with the observation and conceptualization (step 1) of the structure from which an a-priori model is developed in order to design in-situ experiments. The data collected is then processed and used to identify the system for subsequent prediction by simulation (step 6). In spite of the original intention by Moon and Aktan [32] for step 6 to iterate back to step 1 , this methodology does not fully reflect the iterative aspect of data interpretation. Practice has shown that, prior to interpreting measurements, engineers may not fully understand all possible model classes of structural behavior. For complex structures, a multi-stage backtracking procedure is often required because the diagnosis task is an exploratory process involving several iterations [33] of observation and measurements, data interpretation, modeling and performance predictions.

This paper presents a new structural identification framework based on an iterative falsification process and knowledge-based reasoning. This framework is illustrated for the structural identification of a complex bridge structure where several uncertainties related to the structural behavior prevents single pass 
identification. It is demonstrated that the iterative structural identification framework is able to explore compatibility of several model classes of the structure by falsifying inadequate model classes. Thus, this approach is able to make diagnosis and prognosis of the structural conditions using engineering heuristics and on-site measurements, and is robust to modeling systematic uncertainties.

Section 2 describes the iterative structural identification framework along with the tasks to be performed. Section 3 presents the steps of the framework applied to a full-scale bridge and a discussion of the resulting diagnosis. Finally, Section 4 contains a summary and discussion of the iterative aspects and future work.

\section{Iterative structural identification framework}

Structural behavior diagnosis is an ill-defined inverse engineering task that is carried out in open-world conditions and thus, under much uncertainty. For these reasons, such tasks usually lead to multiple explanations for the structural health management of existing structures. The number of possible explanations may be reduced by acquiring knowledge of the structural behavior. The experience and judgment of the engineer as well as other forms of heuristic knowledge are thus of utmost importance. In the field of knowledge-based reasoning, knowledge is acquired by new information obtained using data-interpretation tools [34]. Through these tools, engineers may test their knowledge and their hypotheses against observations.

Diagnosis tasks are usually solved through a process of hypothesis generation and testing. Hypotheses are generated at an early stage from a basic knowledge acquired from limited information. While an early-stage hypothesis may be revised or discarded if subsequent data fail to confirm it, it is likely that at least some hypotheses are correct. Hypotheses are used to organize engineering knowledge and they help to reduce the size of diagnosis task search space. Because it would not be possible to guide an efficient diagnosis task without some hypothetical purpose, hypotheses serve to transform an open-world ill-defined task into a set of well-defined deductive tasks. This process is done iteratively while gradually acquiring knowledge from new observations and from rejected hypotheses.

In this context, the structural identification framework is governed by the principle of falsification, which has been well known by scientists for centuries. However, this principle has only been popularized in the 1930's by Popper [35]. His philosophy stipulates that hypotheses cannot be fully validated by observations and rather can only be falsified by observations. Several authors, such as Tarantola [36], Beven [37] and Beck [26], underlined the advantages of this philosophy since it avoids biasing observations by hypotheses.

For structural identification, hypotheses are usually represented by models and observations by behavior measurements. In addition, basic knowledge is composed of experience, such as information acquired by structural drawings and other inspection reports that may have been established during the service life of the structure, and this serves to develop early-stage hypotheses. Combining structural mechanics theory with such basic knowledge is not usually sufficient for complete definition of the model class. Thus, it is 


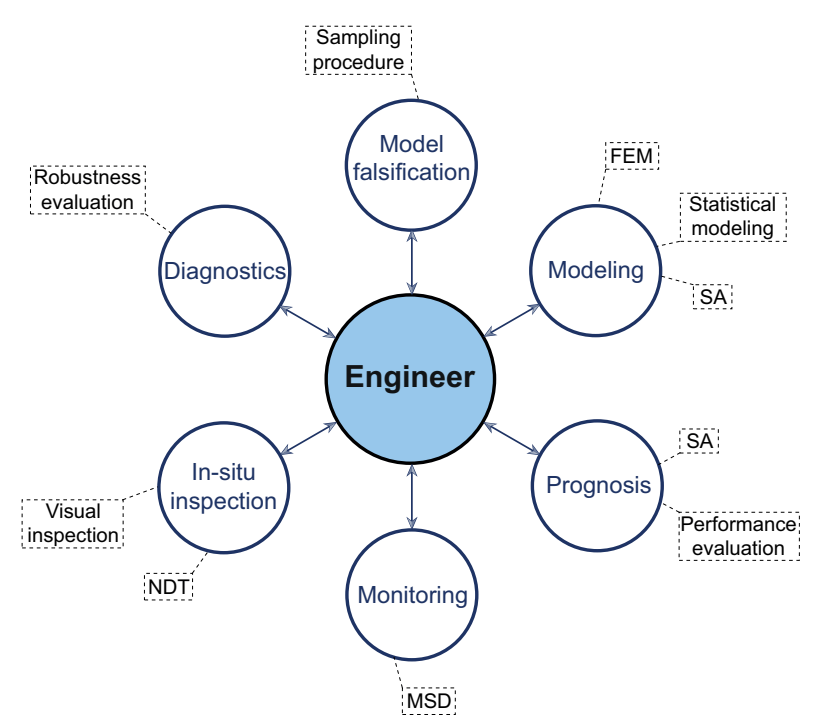

Figure 1: Iterative structural identification framework showing tasks in circles and data-interpretation tools in rectangles. Finite-element model (FEM), sensitivity analysis (SA), measurement-system design (MSD) and nondestructive testing (NDT).

often necessary to return to previous steps in order to iteratively converge upon a correct diagnosis using several model classes.

In Figure 1, an iterative identification process is illustrated where the engineer is in the center of the process. Six tasks are necessary for supporting engineers; modeling, in-situ inspection, monitoring, model falsification, diagnostics and prognosis. Engineers start anywhere and at any stage, and they might go back to previous steps. Tasks are carried out iteratively and the direction taken for the next step is based on engineering decisions based on either the information available at the current step or their knowledge. Data-interpretation tools are available to help engineers solve each task.

The operating principles of this framework are:

- The process is guided by engineers who perform tasks and decide the next tasks to perform based on their knowledge and information acquired in current and previous tasks. It is likely that tasks are performed several times.

- Based on the principle of parsimony (Ockham's razor), simpler model classes are preferred over more complicated classes.

- Model-class falsification may lead to the conclusion that modeling assumptions are not compatible with observed behavior. Especially after decades of service life, structures may behave in a complex manner because of degradation of elements. In such situations, it is important to explore a range of 
model classes that might explain observed behavior. This exploratory approach allows the rejection of an entire class of models. It is thus possible to explore the compatibility of several model classes with observations. In situations where several model classes are compatible with observations, the engineer may compare the performance of each model class and decide to use one or more model classes to support decision making.

- Following iteration, diagnostics and prognosis are typical terminal tasks. Thus, after these tasks, robustness of the results and future performance of the structure are evaluated.

\subsection{Modeling task}

The modeling task consists of building a physics-based model that describes the structural behavior and a statistical model of the errors associated with the physics-based model. For the physics-based model, finiteelement (FE) models are most suitable for representing the behavior of complex structures. In addition, such models are usually associated with uncertainties caused by unknown physical properties, simplifications and omissions. Among the sources of modeling uncertainties, some may be parametrized and then identified by comparing them with measurements.

Other sources of uncertainty may be avoided by including them explicitly in the FE model; for example, components such as reinforcement bars in concrete decks, barriers, diaphragms, etc. Some sources however cannot be included in the FE model either due to the time spent modeling them or due to the computational demand in the FE analysis. These uncertainties may be either of random or of systematic nature. If not included in the FE model, these sources of uncertainty lead to model-prediction errors that should be taken into account when comparing predicted and measured responses. For this reason, errors associated with the simplifications of the physics-based model are estimated for each measurement location using statistical models. Special care is taken when modeling systematic uncertainties since they are usually not centered on zero and are non-Gaussian [38]. Since the statistical model of modeling errors is related to the physics-based model, both models define the model class.

Parametrized variables are usually uncertain material and geometrical properties as well as stiffness of boundary conditions and connections. Among these variables, some have more influence than others on the structural response. Sensitivity analysis (SA) tools may be used to distinguish the importance of these variables in order to select those having the most importance to be identified [39, 40]. The number of parameters to be identified is limited by the computational demand required in following tasks. However, several full-scale examples of civil structures have shown that the number of parameters is usually less than ten $[41-43]$. 


\subsection{In-situ inspection task}

In-situ inspection comprises visual inspection and other nondestructive testing (NDT) techniques. This task includes comparing basic knowledge of the real structure with results of inspection and thus, collecting information on site that would not be on structural drawings and previous inspection reports. Deterioration may be detected and initial knowledge of physical properties may be revised. The engineer may then modify the model class based on this information. Also, NDT techniques may provide information on material properties that refine the estimation of initial model-parameter ranges and thus, help to refine the results.

\subsection{Monitoring task}

Measuring the response, either static or dynamic, at judicious locations allows engineers to test hypotheses related to the structural behavior. Measurements are able to falsify incorrect model classes in order to uncover erroneous assumptions. They can also reduce the uncertainty associated with diagnostics and prognosis tasks [43].

As shown by Goulet and Smith [44], more measurements does not mean higher performance of structural identification. Indeed, they demonstrated that over-instrumentation appears when the new information that is provided by additional measurements is exceeded by the amount of uncertainty provided by the additional measurements. In addition, Pasquier et al. [45] argued that the higher the number of measurements used for structural identification, the greater the probability of making a diagnostic error in the case of misevaluation of modeling uncertainties.

Measurement-system-design (MSD) strategies such as [44, 46] may be used to guide the choice of measurement locations. However, redundancy in the monitored locations is required in order to prevent the effects of erroneous measurements. In addition, when performing static load tests, it is preferable to take measurements for several load configurations in order to increase information related to the structural behavior.

This task also involves the choice of a subset of measurements to be compared with model predictions. Subsets of measurements are usually used in a first step to limit computational demand for preliminary comparison. As knowledge is acquired, the size of measurement sets usually increase.

\subsection{Model falsification task: error-domain model falsification}

Proposed by Goulet and Smith [3], the error-domain model falsification approach aims to obtain possible values for $\boldsymbol{\theta}=\left[\theta_{1}, \ldots, \theta_{n_{\theta}}\right]^{\top}$, describing a vector of $n_{\theta}$ parameter values of a physics-based model using information provided by measurements. Model parameters describe material, physical and geometrical properties of a structure. Estimates for $i=1, \ldots, n_{y}$ characteristic responses $Y_{i}$ of a structure can be provided by models as well as by behavior measurements. Let $g_{\kappa}\left(x_{i}, \boldsymbol{\theta}_{\kappa}\right)$ denote model predictions from a model class $\mathcal{G}_{\kappa}$ and take as input the locations of the predicted degrees of freedom $x_{i}$ and a set of random 
variables $\boldsymbol{\Theta}_{\kappa}$ describing parameter values $\boldsymbol{\theta}_{\kappa}, \hat{y}_{i}$ denotes observations, and $\left\{U_{i, g_{\kappa}}, U_{i, \hat{y}}\right\}$ respectively denotes random variables describing model-prediction and measurement errors for the $i^{\text {th }}$ structural characteristic response. The relationship between a characteristic response and a model prediction is given by

$$
Y_{i}=g_{\kappa}\left(x_{i}, \boldsymbol{\Theta}_{\kappa}\right)+U_{i, g_{\kappa}}, \quad \forall i=1,2, \ldots, n_{y}
$$

and between a characteristic response and a measurement is

$$
Y_{i}=\hat{y}_{i}+U_{i, \hat{y}}, \quad \forall i=1,2, \ldots, n_{y}
$$

The joint PDF $f_{\mathbf{U}_{\hat{y}}}\left(\mathbf{u}_{\hat{y}}\right)$ describing the measurement error is in common cases estimated from repeated calibration experiments performed in controlled conditions. In the case of civil structures, such estimation is usually not possible for the joint PDF of model-prediction errors, $f_{\mathbf{U}_{g_{\kappa}}}\left(\mathbf{u}_{g_{\kappa}}\right)$; instead, $f_{\mathbf{U}_{g_{\kappa}}}\left(\mathbf{u}_{g_{\kappa}}\right)$ is commonly estimated based on heuristics and engineering experience, including systematic errors. Examples of sources of modeling uncertainty are idealized support and connection conditions, temperature effects, load amplitude and load position, Bernoulli-beam hypothesis, geometric variability of the structure, constitutive law of materials, etc. For FE models, examples are also mesh refinement and interpolation, element-type choices, the presence of singularities, etc. Since modeling uncertainty associated with complex civil structures commonly has a larger variance than measurement uncertainty, the joint PDF describing the combination of modeling and measurement uncertainties, $f_{\mathbf{U}_{\mathrm{c}}}\left(\mathbf{u}_{\mathrm{c}}\right) \sim \mathbf{U}_{\hat{y}}-\mathbf{U}_{g_{\kappa}}$ is also dominated by heuristics and engineering experience.

Error-domain model falsification performs structural identification by generating an initial set of $n_{\Omega}$ model instances $\Omega_{\kappa}=\left\{\boldsymbol{\Theta}_{\kappa, m}, m=1,2, \ldots, n_{\Omega}\right\}$ of a model class $\mathcal{G}_{\kappa}$ and then falsifies instances that are not compatible with observations given measurement uncertainties and modeling uncertainties associated with the model class $\mathcal{G}_{\kappa}$. The candidate model set $\Omega_{\kappa}^{*}$ consists of the initial model set minus the falsified models so that

$$
\Omega_{\kappa}^{*}=\left\{\boldsymbol{\theta}_{\kappa} \in \Omega_{\kappa}: u_{i, \text { low }} \leq g\left(x_{i}, \boldsymbol{\theta}_{\kappa}\right)-\hat{y}_{i} \leq u_{i, \mathrm{high}}, \forall i\right\}
$$

where $u_{i, \text { low }}$ and $u_{i, \text { high }}$ are threshold bounds defining the shortest intervals including a probability $\phi_{d}^{1 / n_{y}}$ for the marginal PDFs of $f_{\mathbf{U}_{c}}\left(\mathbf{u}_{c}\right)$, where $\phi_{d} \in[0,1]$ is the target identification reliability usually set at 0.95 . In addition, the number of model instances in the candidate-model set is $n_{\Omega^{*}}$ and each instance is equally likely to be the correct representation of the structure.

All model instances that have been falsified are assigned a probability of 0 so that

$$
\operatorname{Pr}\left(\boldsymbol{\Theta}_{\kappa}=\boldsymbol{\theta}_{\kappa} \notin \Omega_{\kappa}^{*}\right)=0
$$

and all model instances belonging to the candidate-model set are assigned a constant probability

$$
\operatorname{Pr}\left(\boldsymbol{\Theta}_{\kappa}=\boldsymbol{\theta}_{\kappa} \in \Omega_{\kappa}^{*}\right)=\frac{1}{\int_{\boldsymbol{\theta}_{\kappa} \in \Omega_{\kappa}^{*}} \mathrm{~d} \boldsymbol{\theta}_{\kappa}}
$$




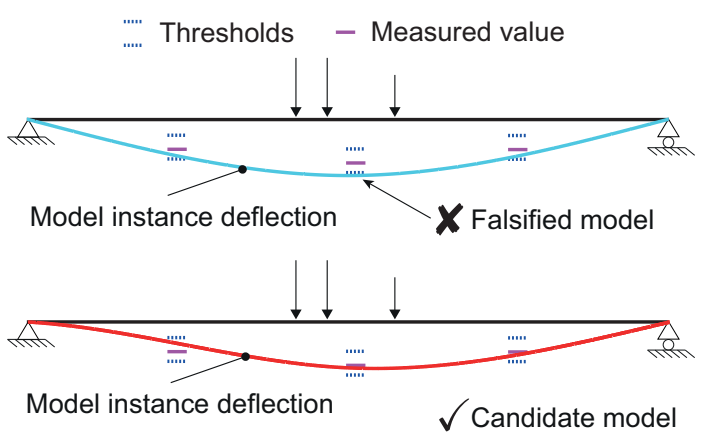

(a)

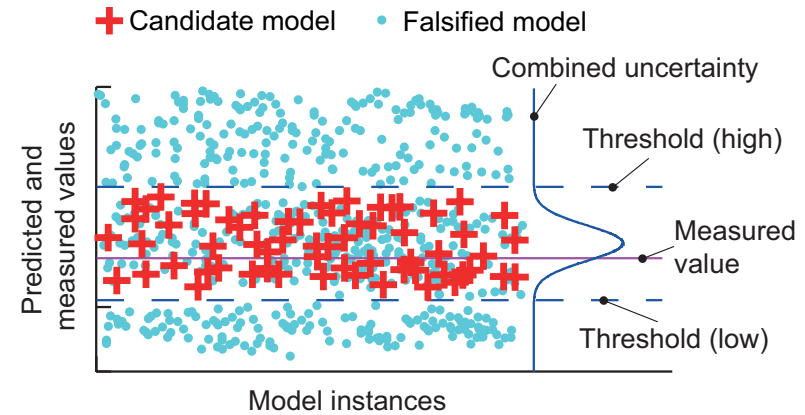

(b)

Figure 2: Model falsification example for a simple beam. (a) Model-instance falsification and acceptance using three measurements. (b) Sensor plot describing the falsification of a set of model instances for a single measurement location. Falsified model predictions that lay within the threshold bounds are predictions of model instances that are falsified at other measurement locations.

Figure 2a presents examples of falsification and acceptance of a model instance for a simple beam based on three measurement locations. A model instance which predictions do not lay within the threshold bounds for the three locations is falsified. A model instance with a different combination of parameter values which predictions lay within the thresholds for every location is a candidate model. Figure $2 \mathrm{~b}$ shows a sensor plot which compares a single measured value with the predictions of the initial model set and the threshold bounds. Candidate-model predictions are within the thresholds. Falsified model predictions that lay within the threshold bounds are predictions of model instances that are falsified at other measurement locations.

In some cases, it is possible that EDMF leads to $\forall \boldsymbol{\theta}_{\kappa}: \operatorname{Pr}\left(\boldsymbol{\Theta}_{\kappa}=\boldsymbol{\theta}_{\kappa}\right)=0$ which results in the complete falsification of the initial population of model instances, $\Omega_{\kappa}^{*}=\emptyset$. This means that there is a likely error in assumptions that led to model-class building and thus, the model class $\mathcal{G}_{\kappa}$ is falsified. Such diagnosis guides the engineer to search for erroneous assumptions and to explore alternative model classes that may be compatible with the set of measurements.

In practice, a sensitivity analysis is carried out during the modeling task (see Section 2.1) to determine the relative importance of each uncertainty source. The uncertainty of the model response at the measured locations due to the parametrized sources are evaluated through Monte Carlo sampling and the FE model. Parametrized sources that have the major importance are included in the primary parameter vector $\boldsymbol{\theta}_{\kappa}$ and are used to generate the initial model set $\Omega_{\kappa}$. Parametrized sources with minor importance are included in the secondary parameter vector $\gamma_{\kappa}=\left[\gamma_{1}, \gamma_{2}, \ldots, \gamma_{n_{\gamma}}\right]^{\top}$. These secondary parameters induce modeling uncertainties $\mathbf{V}_{g_{\kappa}}$ that should be taken into account when comparing model predictions with measurements. The secondary parameter uncertainties are given by

$$
\mathbf{V}_{g_{\kappa}}=g\left(\mathbf{x}, \overline{\boldsymbol{\theta}}_{\kappa}, \boldsymbol{\Gamma}_{\kappa}\right)-g\left(\mathbf{x}, \overline{\boldsymbol{\theta}}_{\kappa}, \overline{\boldsymbol{\gamma}}_{\kappa}\right)
$$


where secondary parameters $\gamma_{\kappa}$ are described by random variables $\boldsymbol{\Gamma}_{\kappa}$, and $\overline{\boldsymbol{\theta}}_{\kappa}$ and $\bar{\gamma}_{\kappa}$ are mean values of $\boldsymbol{\Theta}_{\kappa}$ and $\boldsymbol{\Gamma}_{\kappa}$.

Other sources of uncertainties, $\mathbf{W}_{g_{\kappa}}$, are estimated based on heuristics and engineering experience as presented in Section 2.1. $\mathbf{V}_{g_{\kappa}}$ is added to the other modeling uncertainties $\mathbf{W}_{g_{\kappa}}$, such that the combined uncertainties

$$
\mathbf{U}_{\mathrm{c}}=\mathbf{U}_{\hat{y}}-\mathbf{U}_{g_{\kappa}}=\mathbf{U}_{\hat{y}}-\left(\mathbf{V}_{g_{\kappa}}+\mathbf{W}_{g_{\kappa}}\right)
$$

In this way, systematic and zero-mean random modeling uncertainties are included in the model-falsification process. Note that usually, the simpler the FE model, the greater the variance of the modeling uncertainty $\mathbf{U}_{g_{\kappa}}$, which cannot be reduced using model falsification. Conversely, the random uncertainty associated with primary parameters $\boldsymbol{\theta}$ is reduced by the information provided by measurements.

Several sampling techniques are available in order to generate the initial model set $\Omega_{\kappa}$. Depending on the number of parameters $n_{\theta}$ and precision of the identified parameter values, either uniform sampling or Latin hypercube sampling (LHS) is used. If necessary, Markov Chain Monte Carlo sampling [18] may be used in situations where LHS leads to excessive computation times. Structural identification of existing bridges commonly necessitates less than ten parameters. Whatever sampling method is used, the initial distribution of the parameter values is usually uniform with bounds determined based on engineering judgment and depending on the nature of the physical parameter.

\subsection{Diagnostics task}

The diagnostics is the task that makes sense of the identification results of physical properties of the structure and leads to conclusions about the structural conditions. When the model class is compatible with the set of measurements, a candidate model set is identified, $\Omega_{\kappa}^{*} \neq \emptyset$, and candidate parameter values are determined. This solution may be used to confirm assumptions about the structural behavior that were made during the modeling task and thus increase the knowledge of the structural behavior.

Robustness-evaluation techniques may be used to determine the diagnostic sensitivity to conditions such as misevaluation of uncertainties and changes in correlations between measurements at different measurement locations. These tools help minimize the false positive and false negative diagnostics either at the model-class or the model-instance level.

For example, when performing structural identification with a high number of sensors and several load cases, some measurements may have faulty behavior during load tests. These measurements may not be detected using engineering common sense and outlier detection procedures. However, these measurements may either bias the diagnostics or wrongly falsify a correct model class. A study of the sensitivity to erroneous measurements is thus conducted in order to evaluate diagnosis robustness. For this purpose, it is proposed to carry out model falsification iteratively by removing individually each measurement under any load case. Thus, $n_{y}-1$ measurements are compared with model predictions for each iteration. In a 
second step, each measurement is removed one by one for every load case since it is likely that an erroneous measurement remains erroneous during other load cases. During the two processes, the number of candidate models obtained for each iteration is stored and the sensitivity to erroneous measurements can be evaluated through the variation in the number of candidate models.

\subsection{Prognosis task}

This task involves using identification results for predicting quantities under conditions other than those prevailing during monitoring, for example, model extrapolation for other load configurations [14]. An example in the field of civil engineering is prediction of remaining fatigue life of bridge critical details under traffic loads [43]. The results of prognosis tasks lead to important knowledge necessary for decision making involving retrofit, repair and replacement of existing structures. In this framework, predictions are performed based on the candidate models obtained using EDMF. The prediction of a quantity $q_{j}$ at $n_{q}$ locations of a structure is given by

$$
Q_{j}=g\left(x_{j}, \boldsymbol{\Theta}_{\kappa}^{*}\right)+U_{j, g_{\kappa}}, \quad \forall j=1, \ldots, n_{q}
$$

in agreement with Eq. (1), where $\boldsymbol{\Theta}_{\kappa}^{*}$ is described by the PDF

$$
f_{\boldsymbol{\Theta}_{\kappa}^{*}}\left(\boldsymbol{\theta}_{\kappa}\right)= \begin{cases}\frac{1}{\int_{\Omega_{\kappa}^{*}} \mathrm{~d} \boldsymbol{\theta}_{\kappa}}, & \text { if } \boldsymbol{\theta}_{\kappa} \in \Omega_{\kappa}^{*} \\ 0, & \text { otherwise }\end{cases}
$$

that is based on Eq. (4) and (5). Thus, $Q_{j}$ is a random variable describing the distribution of the predicted quantity $q_{j}$ that is obtained by the combination of the predictions of random candidate-model instances and the distribution of modeling uncertainties associated with model class $\mathcal{G}_{\kappa}$ at the $j^{\text {th }}$ prediction location, $U_{j, g_{\kappa}}$. The lower and the higher threshold bounds of $Q_{j}$ are then determined based on the target prediction reliability $\phi_{p} \in[0,1]$ usually set at 0.95 . They define the shortest intervals including a probability $\phi_{p}^{1 / n_{q}}$ for each PDF $Q_{j}$. Consecutively, since the identification reliability is $\phi_{d}$, the probability of having the true prediction value included between prediction thresholds for each location is at least $\phi_{d} \cdot \phi_{p}$, given the estimated PDF of uncertainty.

Prognosis performance evaluation may be carried out in order to determine whether or not the uncertainty associated with the predicted quantity is acceptable for making good decisions regarding the management of the structure. In situations where the prediction is too uncertain, sensitivity analysis may be used to identify the main uncertainty sources and guide the engineer in the next iteration of the framework. Examples of next steps are monitoring at locations that are related to high uncertainty sources and intervention in order to either repair or replace bridge components that contribute to uncertainty.

\subsection{Framework summary and example}

The proposed framework supports structural identification based on an iterative falsification process and reasoning with engineering knowledge. This approach is suitable for ill-defined tasks such as structural 
identification where information is obtained gradually. The engineer is at the core of the process and makes decisions regarding next steps among the six tasks in order to increase knowledge of structural behavior.

An example of this process is presented in Figure 3 where arrows describe engineering decisions. It starts with an in-situ inspection, continues with the model-class modeling based on structural drawings, then monitoring of judicious locations and model falsification using these measurements. In this case, the model class is falsified due to erroneous assumptions in the preliminary modeling task and thus no diagnostics is provided. The engineer decides to further inspect the structure in order to understand the cause of modelclass falsification. After correction of the model class, the model falsification returns candidate models from which structural diagnosis is possible. In the last step, a prognosis can be made in the scope of structural management decision making. If the performance evaluated in the prognosis step is adequate, the process ends and the engineer makes a decision. In the case where the prognosis is too uncertain, the engineer may decide to perform additional structural monitoring, focusing on the locations that induce high behavior uncertainty.

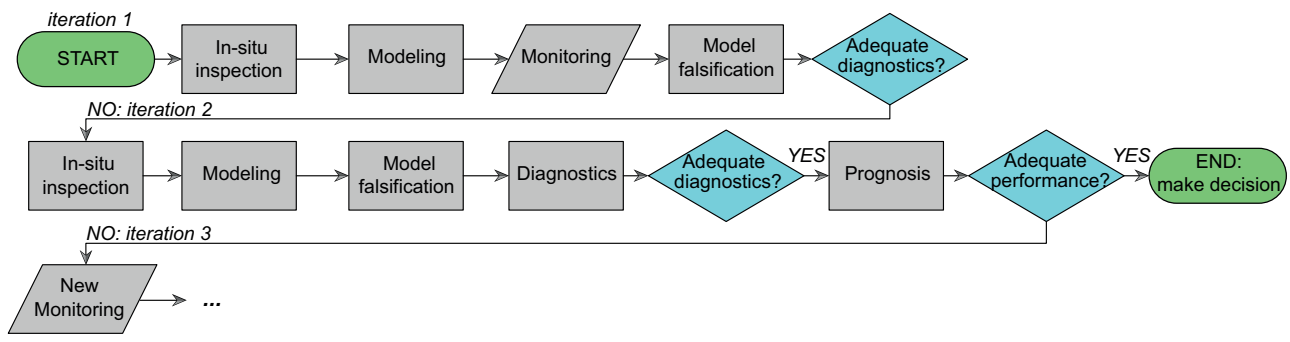

Figure 3: Iterative structural identification process example.

\section{Case study: full-scale bridge in Wayne, New Jersey}

This thirty-year-old bridge is part of US202/NJ23 through Wayne, in New Jersey (USA). The bridge carries eight traffic lanes over four simply-supported spans. The focus is on the second southbound span four lanes that are displayed in Figure 4a. This bridge span has eight steel girders acting in a composite manner with a concrete deck (see Figure 4b). The span geometry is skewed and slightly curved in elevation. The straight-side girders are supported by eight fixed bearing devices and the skewed-side girders are supported by expansion bearing devices. A static load test is carried out using several truck load-case configurations and measuring, for each configuration, twelve vertical displacements (see Figure 5).

Displacement sensors were positioned in a grid manner on the bottom flanges of girders 1, 3,6 and 8 at the quarter, half and three quarter spans. Load configurations of LC-1 to LC-3 consisted of three full trucks positioned on three lanes at the quarter, half and three quarter spans. The fourth lane was open to traffic 


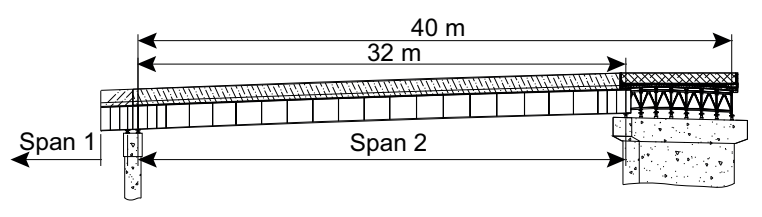

(a)

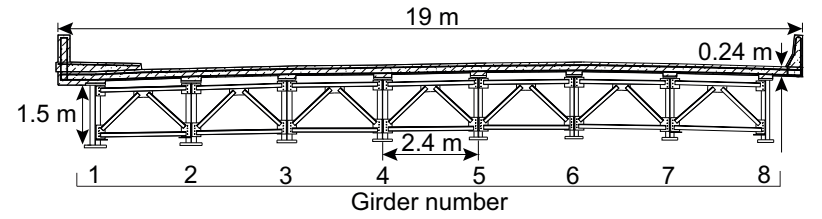

(b)

Figure 4: (a) Bridge elevation view; (b) bridge cross-section view (Adapted from [38]).
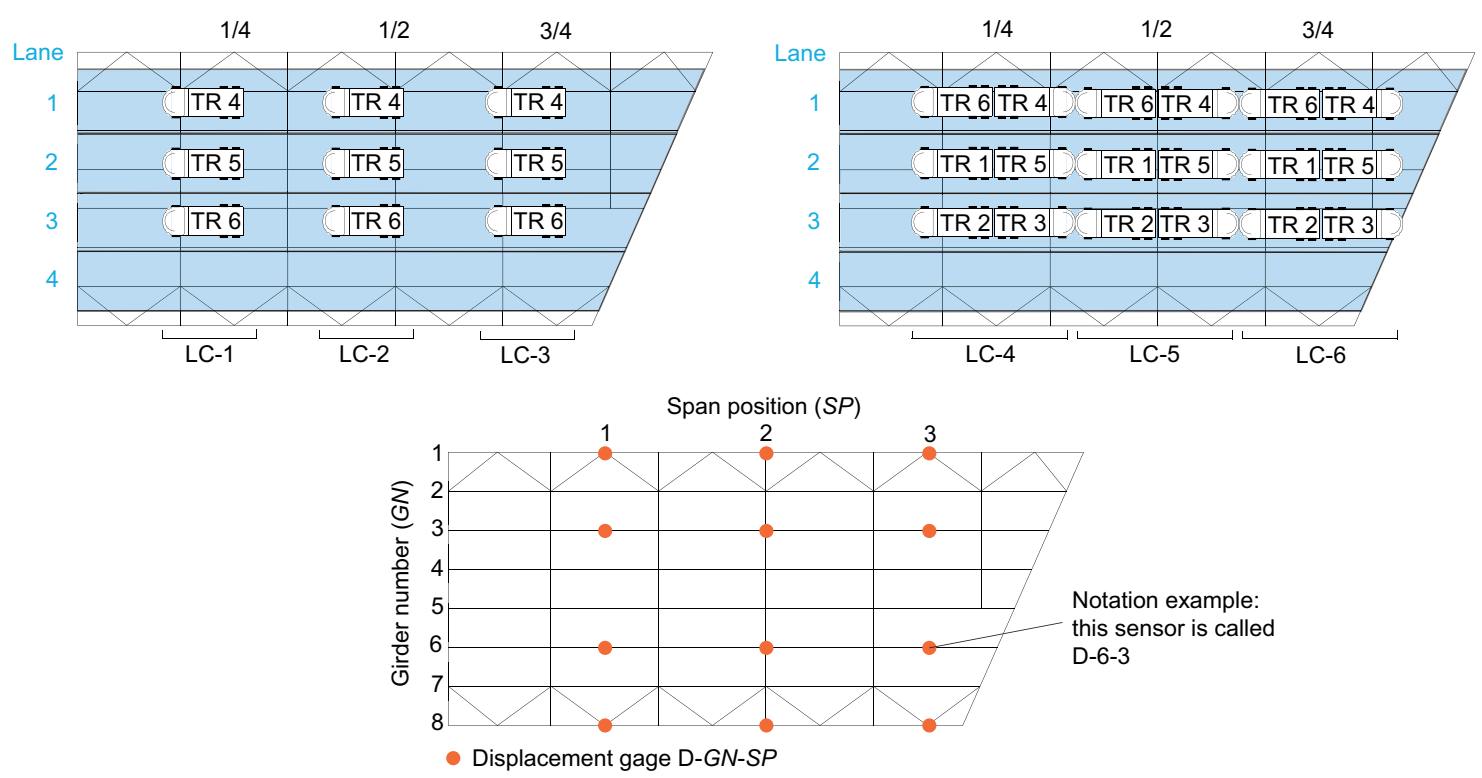

Figure 5: Configurations of static-load tests and displacement gages: six load cases (LC) using six trucks (TR) and twelve displacement gages named as D- $G N-S P$. 
during the static load test. For LC-4 to LC-6, the position was similar and the configurations involve six full trucks weighing around double of the load in LC-1 to LC-3.

Structural identification is carried out using four load cases (LC-1, 2, 3 and 5) and the twelve vertical displacements as shown in Figure 5. Load cases LC-4 and LC-6 are kept for the verification of the identification results. This bridge is a typical example of an aging structure that needs better management. The objective of structural identification is to make a diagnosis for structural health management and discuss the possibility of making a prognosis. The iterative structural identification framework presented in Section 2 is used to perform this task.

\subsection{Identification framework iteration 1}

The structural identification framework first iteration starts with the monitoring phase where the full data set is acquired. The next step of the framework is modeling using knowledge acquired from structural drawings and engineering experience. The engineer decides to build a FE model. When modeling such a complex structure, several assumptions are made and these result in modeling uncertainties. Some sources of modeling error have a random nature while other sources are systematic; they are caused by aspects such as inappropriate model forms and simplified boundary conditions. When the FE model does not account for a source of uncertainty, the modeling uncertainty induced has to be taken into account when comparing model predictions with measurements. Thus, the more aspects included in the FE model, the less uncertainties to be taken into account for the model falsification task and the better performance obtained for structural identification.

For the performance of structural identification, a detailed 3D FE model whose model predictions are described by $g_{1}(\mathbf{x}, \cdot)$ is built. The rows of matrix $\mathbf{x}=x_{k l}$ represents the prediction locations $k \in\{1,2, \ldots, 12\}$ related to the measured locations and the columns, the load cases $l \in\{1,2, \ldots, 6\}$ under which the predictions are calculated. Each element in $x_{k l}$ refers to a measurement location $i$ in Section 2.4 and the number of elements is thus equal to the number of measurements $n_{y}$.

This model includes the concrete deck with reinforcement bars, concrete barriers and the sidewalk, windbraces, diaphragms and stiffeners as shown in Figure 6. Also, special care is taken to include the bridge curvature, distributed truck-wheel loads instead of ideal point loads and the support eccentricity to the cross-section center (see Figure 7 and 8).

For the connection of the girder bottom flange and the bearing pin, rigid links are used such that the rotation of the girder is rigidly transmitted to the pin. In order to allow the longitudinal displacement of the expansion bearing, a pinned rigid link connects the central pin to the bottom bearing. By releasing only the rotational degree of freedom of the bottom pin, the displacement of the central pin is possible. Since friction may exist in the pin, rotational springs are modeled with unknown rotational stiffness parameter values $\gamma_{\text {rot }}$. 


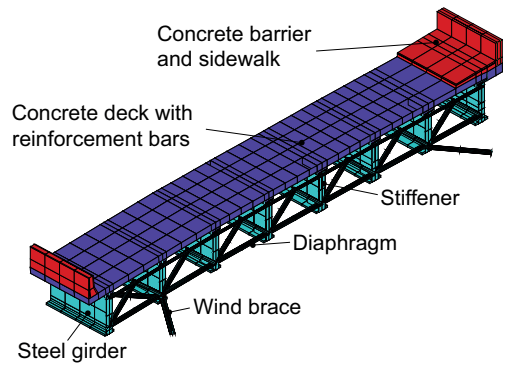

Figure 6: Bridge finite-element model.

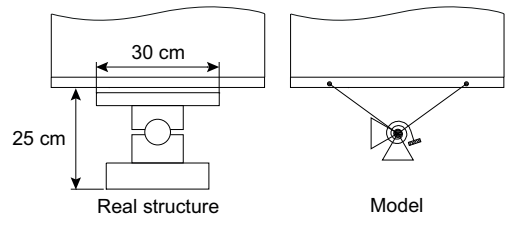

Figure 7: Real and modeled fixed bearing device.

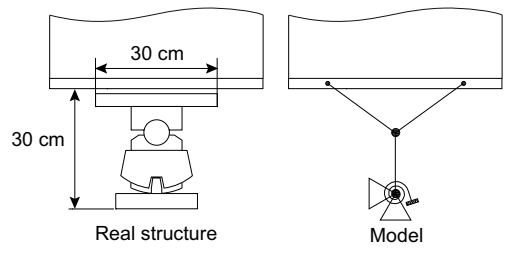

Figure 8: Real and modeled expansion bearing device. 
Table 1: Sources, probability density functions and relative importances of modeling and measurement uncertainties for identification framework iteration 1. PDFs of sources \#1 to \#7 are related to their parameter values. PDFs of sources \#8 to \#15 are associated with characteristic responses. The relative importance values are averaged over all measurement locations.

\begin{tabular}{|c|c|c|c|c|c|}
\hline Uncertainty source & $\mathrm{PDF}$ & Unit & Mean/Min & $\mathrm{SD} / \mathrm{Max}$ & Relative importance \\
\hline 1. Rotational stiffness of bearings & log-uniform & $\log (\mathrm{Nmm} / \mathrm{rad})$ & 6 & 12 & $47 \%$ \\
\hline 2. Young's modulus of concrete & Gaussian & GPa & 21.5 & 4.5 & $16 \%$ \\
\hline 3. Young's modulus of steel & Gaussian & $\mathrm{GPa}$ & 200 & 5 & $3.4 \%$ \\
\hline 4. $\Delta \nu$ Poisson's ratio of concrete & Gaussian $^{\mathrm{a}}$ & - & 0 & 0.025 & $2.4 \%$ \\
\hline 5. $\Delta T$ concrete deck thickness & Gaussian & $\%$ & 0 & 2.5 & $1.3 \% \mathbf{I}$ \\
\hline 6. $\Delta t$ steel plate thickness & Gaussian & $\%$ & 0 & 1 & $1.9 \%$ । \\
\hline 7. $\Delta w$ truck load per wheel & Gaussian & $\mathrm{N}$ & 0 & 225 & $0.7 \% \mathrm{I}$ \\
\hline 8. Simplifications and FEM & uniform & $\%$ & -8 & 1 & $4.3 \% \square$ \\
\hline 9. Mesh refinement & uniform & $\%$ & -1 & 0 & $0.9 \%$ I \\
\hline 10. Truck position & Gaussian & $\%$ & -1 & 0.8 & $2.7 \%$ \\
\hline 11. Sensor resolution & Gaussian & $\mathrm{mm}$ & 0 & 0.13 & $12 \%$ \\
\hline 12. Cable losses & Gaussian & $\%$ & 0 & 0.4 & $1.5 \%$ I \\
\hline 13. Repeatability & Gaussian & $\%$ & 0 & 0.5 & $2.0 \%$ | \\
\hline 14. Traffic noise & Gaussian & $\mathrm{mm}$ & 0 & $0.02-0.1^{\mathrm{b}}$ & $2.7 \%$ 】 \\
\hline 15. Additional uncertainties & Gaussian & $\%$ & -1 & 1 & $1.2 \% \mathbf{I}$ \\
\hline
\end{tabular}

${ }^{\mathrm{a}}$ Gaussian distribution that is truncated at 0.03 and 0.33 .

${ }^{\mathrm{b}}$ Minimum and maximum standard deviation over all measured values.

Uncertainty sources such as material Young's moduli $\left(\gamma_{\text {conc }}\right.$ and $\left.\gamma_{\text {steel }}\right)$, the Poisson's ratio of concrete $\left(\gamma_{\Delta \nu}\right)$, the thickness of the concrete deck $\left(\gamma_{\Delta T}\right)$ and steel plates $\left(\gamma_{\Delta t}\right)$ and truck loads $\left(\gamma_{\Delta w}\right)$ are also parametrized. These sources and their statistical models are displayed in Table 1 from \#1 to \#7. The PDFs describe the uncertainty associated with their parameter value $\gamma$ that is estimated using engineering heuristics and field observations. Their effects on the structural response is then quantified through Monte Carlo simulations using the FE model and Eq. (6).

For the rotational stiffness of bearings, the range of values is evaluated based on the relation between the stiffness value and the displacement responses that are represented in Figure 9. This figure shows that for values lower than $10^{6} \mathrm{Nmm} / \mathrm{rad}$ and values higher than $10^{12} \mathrm{Nmm} / \mathrm{rad}$, the response is not sensitive to the stiffness value. For such ranges of values, the bearing acts either as a pinned or as a fixed support. Thus, the initial significant parameter range is between $10^{6}$ and $10^{12}$ where the parameter value influences the response. 


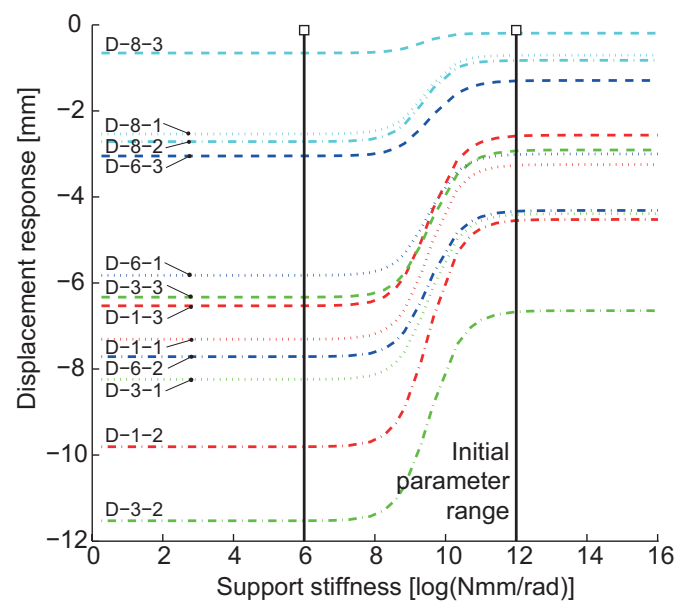

Figure 9: Relation between support rotational stiffness values and displacement responses.

The other sources of uncertainty that cannot be included in the FE model are estimated using engineering judgment with respect to model predictions generated with the mean value of the parameters $\# 1$ to $\# 7$. These sources listed in Table 1 from \#8 to \#10 are model simplifications and FEM idealization $\left(\mathbf{W}_{\text {simp }}\right)$, mesh refinement $\left(\mathbf{W}_{\text {mesh }}\right)$ and truck position $\left(\mathbf{W}_{\mathrm{tp}}\right)$. For source \#8, simplifying assumptions are the non-inclusion of concrete-deck cracks, partially connected barrier, barrier joints that reduce the predicted displacement. In addition, FE models are usually stiffer than reality. Thus, these aspects lead to underestimate displacement predictions. The lower bound $-8 \%$ was determined based on this reasoning and engineering judgment. The non-inclusion of the fence, which increases predicted displacements, explains the value of $+1 \%$ of the upper bound of source \#8. An extensive description of sources and forms of uncertainties is available in [38].

In order to compare model predictions and measurements, measurement uncertainties are also estimated (\#11 to \#15). These sources are sensor resolution $\left(\mathbf{U}_{\text {res }}\right)$, cable losses $\left(\mathbf{U}_{\text {loss }}\right)$, measurement repeatability $\left(\mathbf{U}_{\text {rep }}\right)$ and traffic noise $\left(\mathbf{U}_{\text {traf }}\right)$ during static load tests. Additional uncertainties $\left(\mathbf{U}_{\text {add }}\right)$ are a conservative estimation for all other phenomena that individually have a negligible influence.

Table 1 also presents the relative importance of each uncertainty source on the structural response. This relative importance is averaged over all measurement locations under all load configurations. This shows that the rotational stiffness of bearings is the main source of uncertainty with $47 \%$ of influence. The second most important source is the Young's modulus of concrete with $16 \%$ relative importance. Due to their high influence on the structural response, these two parameters are selected to generate the initial model set. The other parametrized uncertainties have too low of an influence to be identifiable. However, they are included with the remaining uncertainties in $f_{\mathbf{U}_{\mathrm{c}}}\left(\mathbf{u}_{\mathrm{c}}\right)$ as described in Eq. (7) for the calculation of threshold bounds during the falsification process. Note also the high influence of the sensor resolution with $12 \%$ relative importance. 
The first model class $\mathcal{G}_{1}$ is thus composed of $g_{1}\left(x_{k l}, \boldsymbol{\theta}_{1}\right)$ with $\boldsymbol{\theta}_{1}=\left[\theta_{\text {rot }}, \theta_{\text {conc }}\right]^{\top}$ being the rotational stiffness and the Young's modulus of concrete, and the combination of modeling uncertainties \#3 to \#10, $\mathbf{U}_{g_{1}}$ displayed in Table 1 and calculated using Eq. (10).

$$
\mathbf{U}_{g_{1}}=\mathbf{V}_{\text {steel }}+\mathbf{V}_{\Delta \nu}+\mathbf{V}_{\Delta T}+\mathbf{V}_{\Delta t}+\mathbf{V}_{\Delta w}+\mathbf{W}_{\text {simp }}+\mathbf{W}_{\text {mesh }}+\mathbf{W}_{\mathrm{tp}}
$$

Measurement uncertainties are combined based on Eq. (11).

$$
\mathbf{U}_{\hat{y}}=\mathbf{U}_{\mathrm{res}}+\mathbf{U}_{\mathrm{loss}}+\mathbf{U}_{\mathrm{rep}}+\mathbf{U}_{\mathrm{traf}}+\mathbf{U}_{\mathrm{add}}
$$

The rotational stiffness value is the same for all 16 supports since there is no information at this step regarding different stiffnesses of bearing devices. The next step involves the model falsification and the initial model set is generated based on a uniform sampling for which the range given in Table 1 is divided into 11 intervals for both parameters, leading to a set $\Omega_{1}=\left\{\boldsymbol{\Theta}_{1}\right\}$ of $n_{\Omega_{1}}=144$ model instances. Using $n_{y}=24$ displacement measurements of load cases LC-2 and 5 (in $x_{k l}, l \in\{2,5\}$ ). Only a subset of the measurement data set is used to reduce the computing demand of the initial iteration.

This process leads to the complete falsification of the initial model set and thus the falsification of model class $\mathcal{G}_{1}$. As a result, no diagnostic can be provided and it is likely that wrong assumptions led to model-class building.

\subsection{Identification framework iteration 2}

Since at this stage, information is lacking regarding the source of this model-class error, the engineer decides to make in-situ visual inspection for the purpose of comparing his prior assumptions about the structure with the real structure. Thus, a second iteration of the identification framework starts. On site, a penetrating crack is observed on the pier cap under the support of girder 1 on the straight bridge side. This crack may reduce the stiffness of the pier cap and thus the assumption of infinite vertical stiffness of the support is no longer valid. In addition, a severe state of corrosion deterioration is observed on the bearing devices, particularly on the exterior bearing devices, i.e. the supports of girder 1 and 8 . The visual inspection also reveals that the concrete deck is orthotropic by observing a steel deck plate on the lower side of the bridge.

In the next step of the identification process, the engineer decides to include the components observed during in-situ inspection in a new model class $\mathcal{G}_{2}$. The FE model is modified to incorporate the orthotropic deck and a vertical spring with unknown stiffness under the support where the pier cap crack is located. The spring stiffness is another uncertain parameter that adds to the other modeling uncertainties. A similar study of the relation parameter value to displacement response as the one of the rotational spring stiffness (see Figure 9) is undertaken. This study leads to a significantly sensitive range of values between $10^{2}$ and $10^{8} \mathrm{~N} / \mathrm{mm}$. 
In order to include the effect of the severe corrosion of the exterior bearings, two distinct parameters are used to describe either the uncertainty of the averaged stiffness of the exterior bearings (i.e. bearings under girder 1 and 8) or the averaged stiffness of the interior bearings. Although larger stiffness values are expected on the exterior bearing than on the interior's, the range of values of both parameters remains identical as the values displayed in Table 1 . Table 2 presents the sources and the relative importances of uncertainties associated with the identification of model class $\mathcal{G}_{2}$.

Table 2: Sources and relative importance of parameter, modeling and measurement uncertainties for identification framework iteration 2 . The relative importance values are averaged over all displacement measurement locations.

\begin{tabular}{ll}
\hline Uncertainty source & Relative importance \\
\hline Exterior bearing rotational stiffness & $14 \%$ \\
Interior bearing rotational stiffness & $32 \%$ \\
Young's modulus of concrete & $16 \%$ \\
Modeling uncertainties & $18 \%$ \\
Measurement uncertainties & $19 \%$ \\
Pier-cap stiffness & $1.0 \%$ \\
\hline
\end{tabular}

Exterior and interior bearing stiffnesses and the Young's modulus of concrete have a high importance and thus are selected as identification parameters. The pier-cap stiffness uncertainty $\left(\mathbf{V}_{\text {pier }}\right)$ has very low influence on the displacement response and thus is added to modeling uncertainties $\mathbf{U}_{g_{1}}$, which remains in this model class, for the determination of $\mathbf{U}_{g_{2}}$ as described by

$$
\mathbf{U}_{g_{2}}=\mathbf{U}_{g_{1}}+\mathbf{V}_{\text {pier }}
$$

where $\mathbf{U}_{g_{2}}$ is then used for the determination of $f_{\mathbf{U}_{\mathrm{c}}}\left(\mathbf{u}_{\mathrm{c}}\right)$.

As a result, the new model class $\mathcal{G}_{2}$ is composed of $g_{2}\left(x_{k l}, \boldsymbol{\theta}_{2}\right)$ with parameters $\left[\theta_{\text {rot-ext }}, \theta_{\text {rot-int }}, \theta_{\text {conc }}\right]^{\top}$ to identify and the modeling uncertainties $\mathbf{U}_{g_{2}}$ based on Eq. (12). For the next model-falsification step, an initial model set is generated based on a uniform sampling of the three parameters $\boldsymbol{\theta}_{2}$. Each parameter range is divided into 11 intervals leading to an initial set $\Omega_{2}=\left\{\boldsymbol{\Theta}_{2}\right\}$ of $n_{\Omega_{2}}=1,728$ model instances of model class $\mathcal{G}_{2}$. Using the $n_{y}=24$ displacement measurements of LC-2 and 5, no compatibility is found between the model instances and the measurements $\left(\Omega_{2}^{*}=\emptyset\right)$. Model class $\mathcal{G}_{2}$ is also falsified. The diagnostics of iteration 2 is thus inadequate.

In order to locate the source of the erroneous assumptions leading the falsification of the model class, the measurements of LC-1 and 3 are also included in the set of measurements used for model falsification. Then, subsets of the measurement set are used in order to identify candidate models. By successively selecting the 

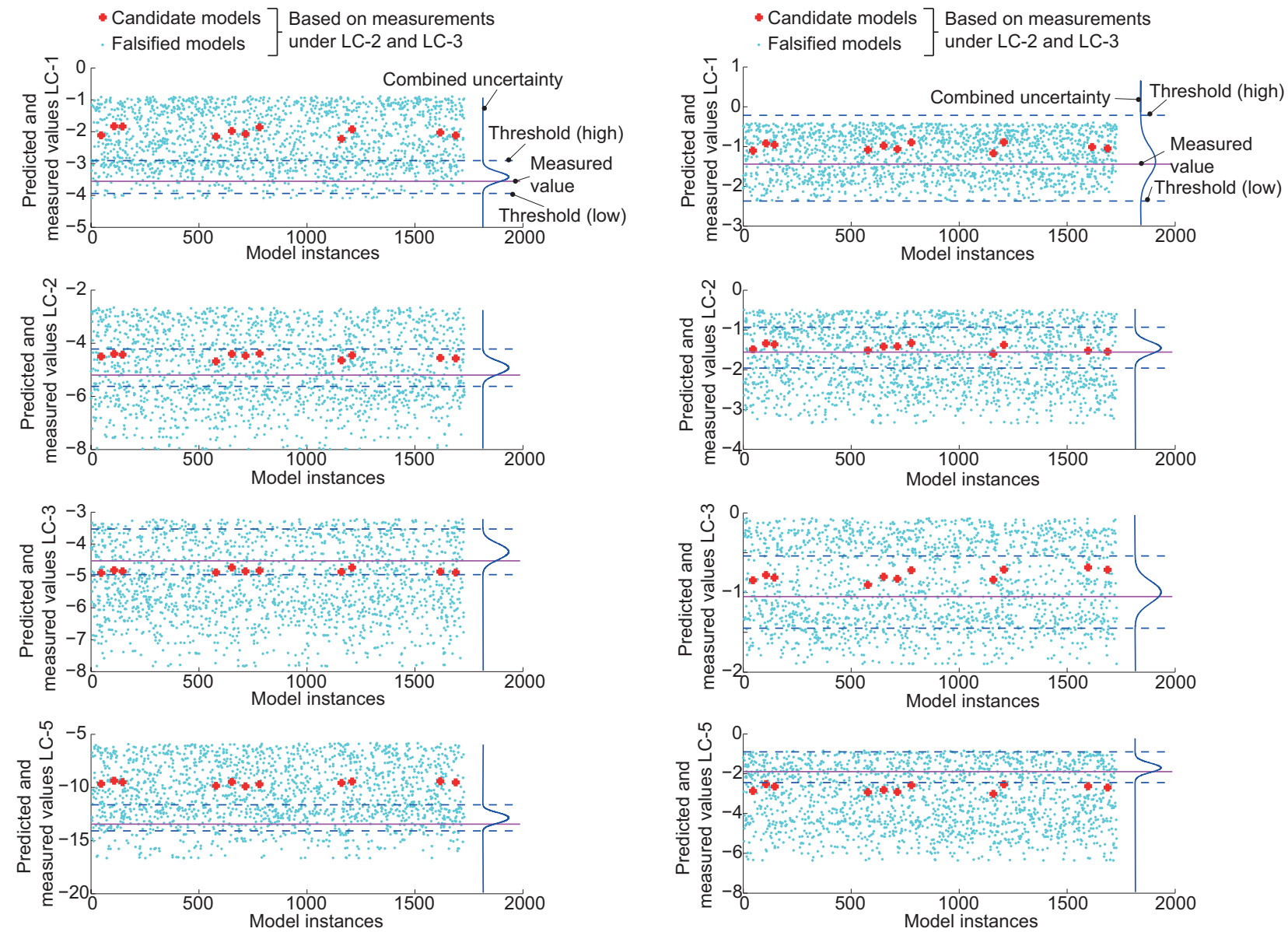

(a)

(b)

Figure 10: Comparison of model predictions and measured values for LC-1, 2, 3 and 5. (a) Sensor D-3-3 ;

(b) Sensor D-8-1.

12 displacements of a single load case, it is observed that only LC-2 and LC-3 are able to identify candidate models. When selected together, these load cases identify 11 candidate models.

Figures 10a and 10b show the initial model predictions (dots) and the eleven candidate-model predictions (crosses) for sensors D-3-3 and D-8-1. In these figures, the measured values, the combined uncertainty and the threshold bounds are also represented. Under LC-2 and LC-3, the candidate-model predictions lay in the region bounded by the thresholds. Predictions that are between the threshold bounds and that are not candidate-model predictions are predictions from model instances that are falsified by the other sensor locations of LC-2 and LC-3.

Candidate-model predictions of LC-1 and LC-5 lay outside the region bounded by the thresholds for sensor D-3-3. These predictions reflect an over-stiff behavior of the candidate models for these locations. Note that this behavior is common to all sensors located on girders 1 and 3. In addition, for sensor D-8-1 
under LC-5, candidate-model predictions reflect that candidate-model instances have a softer response than that which was measured. Note also that this behavior is common to sensor D-8-2. The sensor plot of D-8-1 under LC-1 depicts an example of a redundant sensor, i.e. a sensor that is not able to falsify additional model instances.

These observations may be correlated with the in-situ measurements as a result of the stepwise increasing loading that is used during static load tests. Figure 11 presents the relation between measured displacement values for several sensors and the truck load on the bridge for configurations of trucks positioned at the quarter, half and three quarter spans. This figure involves LC-1 to LC-6 and also empty-truck load cases that not represented in Figure 5 and not used for identification since the amplitude of measured displacement values is not high enough compared with the sensor resolution to be used for structural identification. This
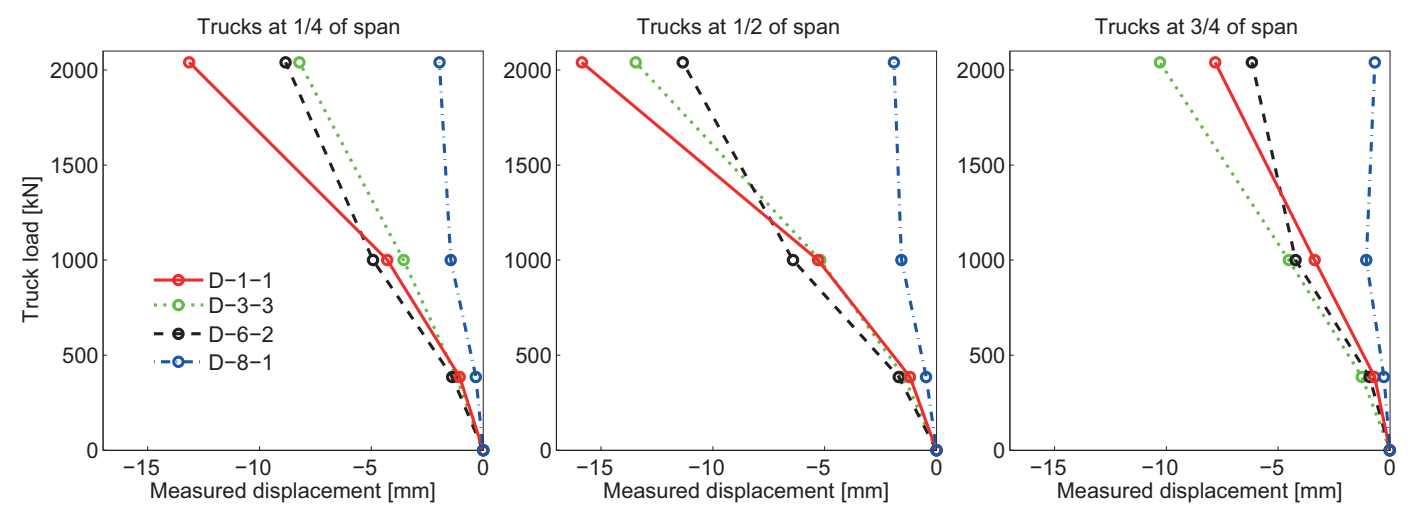

Figure 11: Non-linear relation of displacement responses and truck loads for trucks positioned at the quarter, half and three quarter of the bridge span.

472

figure depicts a non-linear relation between displacement and loading. In addition, for sensors D-1-1 and D-3-3, the behavior is softer as the loading increases and for sensors D-6-2 and D-8-1, the behavior is stiffer in the same conditions. Note that these trends are similar for the majority of measurements. Thus, observations made in Figure 10a and particularly the underestimation of the displacements made by the candidate-model instances is thus caused by the non-linear behavior observed in Figure 11 since the model class $\mathcal{G}_{2}$ assumes a linear behavior under increasing loading conditions. The same correlation can be made between the sensor plot of D-8-1 in Figure 10b and the behavior of displacement values with increasing loading, except that in this case the candidate-model predictions overestimate the displacement values due to the stiffer observed behavior.

From the severe corrosion of the bearing devices that is observed during in-situ inspection and the high influence of the stiffness in the displacement response of the bridge, it can be deduced that non-linear behavior is caused by the bearing devices. Indeed, the non-linear concave behavior observed on girders 1 and 3 may be the result of the corrosion that creates a high rotational stiffness on the support for low loading 
values and this blocking may be released for increasing loads due to increasing moment at support. This behavior does not appear for girders 6 and 8 that have a shorter span than girders 1 and 3 and rather, an increasing load may further block the bearings due to low values of moments in these supports. Based on these observations, the engineer decides to start a third iteration.

\subsection{Identification framework iteration 3}

This new iteration of the identification framework starts with the modeling of non-linear spring behavior of the bearing devices. As more information is not available, it is assumed that the bearings follow a bilinear rotation-moment relation instead of the linear behavior that has been modeled up until this point. Figure 12 shows a schematic description of the bilinear relationship and the unknown parameters $C_{1}, C_{2}$ and $\varphi$ that describe the behavior of the bearings. The parameters $C_{1}$ and $C_{2}$ represent the stiffnesses of the rotational

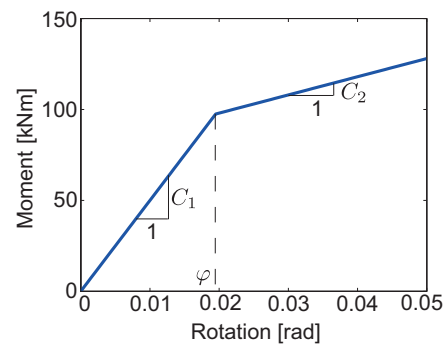

Figure 12: Schematic bilinear model of the moment-rotation relationship of the bearing devices.

springs and $\varphi$ the rotation value for which the stiffness changes from $C_{1}$ to $C_{2}$. Based on the behavior observed in Figure 11, the value of $C_{1}$ may be higher or lower than the value of $C_{2}$ and may be different for each bearing device. Their initial range of values is thus the same as the ranges of rotational stiffness values of model classes $\mathcal{G}_{1}$ and $\mathcal{G}_{2}$ presented in Table 1 . Based on bearing rotation values obtained with the 11 candidate models of the previous falsification, a conservative range of values is estimated for $\varphi$ between 0 and 0.03 radians. Note that a value of 0 rad implies a linear relationship with $C_{2}$ as single parameter.

In order to identify the bearing stiffnesses having the main influence in the bridge response and thus reduce the number of parameters $\boldsymbol{\theta}$, a sensitivity analysis is carried out by varying the stiffness value of all bearings. Table 3 presents the relative importance of the stiffness parameter for each bearing device for the linear FE model. The rotational stiffness of the bearings located on the skewed bridge side show a significantly greater importance than the bearings located on the other side. This means that the influence of a potential non-linear behavior of the straight-side bearing devices is negligible, and thus, they are modeled to be linear.

As a result, the identification parameters of the new model class $\mathcal{G}_{3}$ includes only the stiffness parameters of the skewed side and thus, the unknown parameters of the bilinear model are selected only for these bearings. 
Table 3: Relative importance of rotational stiffness parameter of the 16 bearings. The relative importance values are averaged over all measurement locations. For the stiffness parameters, the number refers to the girder and " $s$ " refers to bridge skewed side.

\begin{tabular}{lll}
\hline Stiffness parameter & \multicolumn{2}{c}{ Relative importance } \\
\hline rot-1 & $1.1 \%$ & $\mathbf{\square}$ \\
rot-2 & $0.8 \%$ & $\mathbf{\square}$ \\
rot-3 & $1.3 \%$ & $\mathbf{\square}$ \\
rot-4 & $1.0 \%$ & $\mathbf{\square}$ \\
rot-5 & $1.8 \%$ & $\mathbf{\square}$ \\
rot-6 & $1.5 \%$ & $\mathbf{\square}$ \\
rot-7 & $1.2 \%$ & $\mathbf{\square}$ \\
rot-8 & $1.7 \%$ & $\mathbf{\square}$ \\
rot-1s & $17 \%$ & \\
rot-2s & $8.4 \%$ & \\
rot-3s & $8.1 \%$ & \\
rot-4s & $8.1 \%$ & \\
rot-5s & $11 \%$ & \\
rot-6s & $14 \%$ & \\
rot-7s & $12 \%$ & \\
rot-8s & $11 \%$ & \\
\hline
\end{tabular}


This reduces the number of parameters to identify from 49 to 25 (i.e. $C_{1}, C_{2}$ and $\varphi$ for each bearing on the skewed side and the Young's modulus of concrete) and $\boldsymbol{\theta}_{3}=\left[\theta_{\text {rot- } 1 \mathrm{~s}-C_{1}}, \theta_{\text {rot- } 1 \mathrm{~s}-C_{2}}, \theta_{\text {rot- }-1 \mathrm{~s}-\phi}, \ldots, \theta_{\text {conc }}\right]^{\top}$. In addition, the modeling uncertainties include the uncertainty associated with stiffness values of linear-behavior bearings rot-1 to rot-8:

$$
\mathbf{U}_{g_{3}}=\mathbf{U}_{g_{2}}+\sum_{r=1}^{8} \mathbf{V}_{\text {rot- } r}
$$

Since the number of parameters is too high to generate the initial model set using uniform sampling, Latinhypercube sampling is employed to generate the initial model set $\Omega_{3}=\left\{\boldsymbol{\Theta}_{3}\right\}$ of $n_{\Omega_{3}}=10,000$ model instances of model class $\mathcal{G}_{3}$. However, in this model-falsification step, all model instances are falsified leading to the rejection of model class $\mathcal{G}_{3}$. This means that the assumed bilinear behavior of the bearings is an erroneous assumption. This implies that the real behavior of the bearing is more complex than this simplified bilinear model. In addition, although the corrosion of the bearings is an important source of non-linear behavior, the geometrical complexity introduced by the skewed bridge side may also add some difficulties when identifying the true behavior of the bearings. Indeed, under some load cases, support reactions under girder 8 appear to be negative (uplift). However, bearings were not designed to accommodate such behavior. Even if the FE model is modified to include this possibility, it is still not sure that the bilinear behavior is a correct assumption. For this reason, the engineer decides to start a fourth iteration of the identification framework with a new model class that includes for the non-linear behavior uncertainty of the bearings as a source of modeling uncertainty.

\subsection{Identification framework iteration 4}

In this fourth iteration, the engineer decides to estimate the uncertainties associated with the non-linear behavior of the bearings in order to include them in a new model class $\mathcal{G}_{4}$. The error due to the non-linear behavior is estimated through the comparison of the measured values and a model that behaves linearly with respect to the loading. Based on the representation of the relationship between measured displacement and truck load, coordinates of points A, B and C are known (see Figure 13). The linear model should pass by

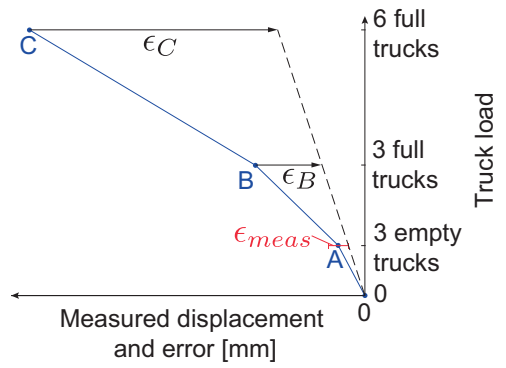

Figure 13: Schematic representation of the estimation of the error associated with the non-linear bridge behavior. 
the coordinates $(0 ; 0)$ and the point $\mathrm{A}$ where the load is caused by the three empty trucks. For this load, the bridge behavior is assumed to be linear. However, since the measured value at point A is associated with a measurement error whose upper bound is $0.25 \mathrm{~mm}$. By adding this upper-bound error value to the measured value in $\mathrm{A}$, a second point is determined in order to draw the linear model that represents the worst case scenario for the error associated with the non-linear behavior. These errors $\epsilon_{B}$ and $\epsilon_{C}$ are then represented by the difference between points $\mathrm{B}$ and $\mathrm{C}$ and the linear model. These values represent an upper bound for the uncertainty. The lower bound is set to 0 and thus the uncertainty associated with the non-linear behavior $\mathbf{W}_{\mathrm{nl}}$ is assumed to follow a uniform PDF with boundaries 0 and either $\epsilon_{B}$ or $\epsilon_{C}$.

With this additional source, the uncertainties associated with model class $\mathcal{G}_{4}$ become

$$
\mathbf{U}_{g_{4}}=\mathbf{U}_{g_{3}}+\mathbf{W}_{\mathrm{nl}}
$$

The FE model is modified in order to account for linear behavior of the bearings as it was for model class $\mathcal{G}_{2}$ such that $g_{4} \equiv g_{2}$. The parameters $\boldsymbol{\theta}_{4}=\left[\theta_{\text {rot-ext }}, \theta_{\text {rot-int }}, \theta_{\text {conc }}\right]^{\top}$ are the rotational stiffness of the exterior bearings, the rotational stiffness of the interior bearings and Young's modulus of concrete. As determined for model class $\mathcal{G}_{3}$, the bearing stiffness of the straight bridge side are included in the uncertainties $\mathbf{U}_{g_{4}}$ due to their low influence on the displacement responses. Thus, only the stiffnesses of bearings located on the skewed side are parameter values requiring identification.

For the next model-falsification step, an initial model set $\Omega_{4}=\left\{\boldsymbol{\Theta}_{4}\right\}$ of $n_{\Omega_{4}}=1,728$ model instances is generated by dividing the parameter ranges to 11 uniform intervals. Using the $n_{y}=48$ measurements of LC$1,2,3$ and 5 and model predictions $g_{4}\left(x_{k l}, \boldsymbol{\Theta}_{4}\right), x_{k l}$ with $k \in\{1,2, \ldots, 12\}$ and $l \in\{1,2,3,5\}, 4$ candidate models are identified and more than $98 \%$ of the initial population is falsified. Although this means that the model class $\mathcal{G}_{4}$ is correct, before making conclusions in the diagnostic step, a study of model-class robustness should be carried out due to the high ratio of rejected models.

\subsubsection{Diagnosis robustness}

The sensitivity to erroneous measurements is conducted in order to evaluate diagnosis robustness as presented in Section 2.5. Thus, in the first step, $n_{y}=47$ measurements corresponding to the removal of a single element in the matrix $x_{k l}$ are compared with model predictions for each model-falsification iteration assuming that only one measurement may be erroneous. In the second step, each measurement is removed one by one for every load case since it is likely that an erroneous measurement remains erroneous during other load cases. Thus, for each falsification iteration, a row is removed from the matrix $x_{k l}$. During the two processes, the number of candidate models obtained for each iteration is stored and the sensitivity to erroneous measurements can be evaluated through the variation in the number of candidate models.

Figure 14 shows the result of both processes for model class $\mathcal{G}_{4}$. When removing individually a single measurement and thus using $n_{y}=47$ measurements, the model falsification leads to the same 4 candidate 

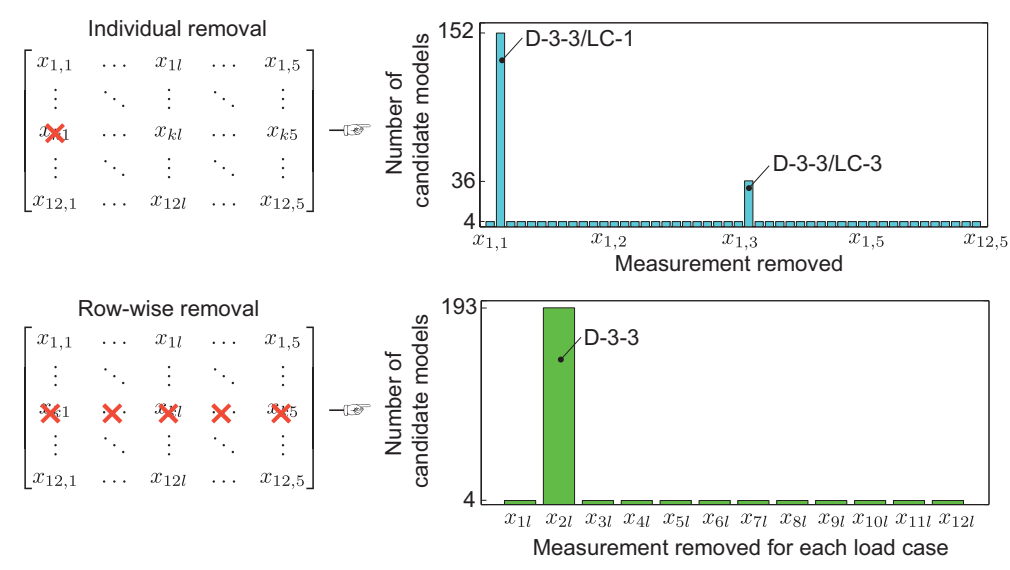

Figure 14: Diagnosis sensitivity to single measurement removal for any load case and for each load case for model class $\mathcal{G}_{4}$.

models as found using the set of 48 measurements, except under removal of $x_{2,1}$ and $x_{2,3}$ measurements referring to sensor D-3-3 under LC-1 and LC-3. The identification results are sensitive to the removal of these measurements since the number of candidate models increases to 152 and 36, respectively. In addition, when removing the measurements for each load case during the model falsification (comparison of $n_{y}=44$ measurements), each measurement configuration obtained leads to the same 4 candidate models except when removing sensor D-3-3 for which the number of candidate models increases to 193.

This investigation shows a high sensitivity of the diagnostics to sensor D-3-3, particularly under LC-1 and LC-3. This means that keeping this sensor in the identification process may hide hundreds of possible solutions. Since the removal of this sensor for two load cases shows diagnostic sensitivity, a robust approach is the removal of this sensor for each load cases and keeping the identification of 193 candidate models as the diagnostic result. In addition, the robust set of 193 candidate models includes the 4 candidate models previously identified.

Figure 15 presents a pairwise comparison of the candidate-model parameter values that are identified for the three parameters. Each axis represents the initial possible values for every parameter. Although this figure shows that the 193 candidate models do not reveal a significant reduction in the parameter ranges, it presents a significant reduction in the number of permutations of the interior bearing stiffness values and concrete Young's modulus values. In addition, the pairwise comparison of exterior and interior bearing values confirms that they are likely unequal due to the difference of their deterioration state.

Note that this sensitivity study is also carried out for model class $\mathcal{G}_{1}$ to $\mathcal{G}_{3}$ in order to guarantee that a single measurement is not responsible for the model-class falsification. Each process returns an empty candidate-model set for any sensor removed ensuring the robustness of the model-class falsification. 


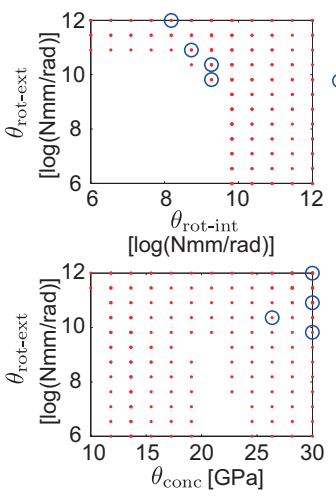

Candidate model

obtained without D-3-3

OCandidate model obtained

using 48 measurements

$\theta_{\text {rot-ext }}$ : Exterior bearing stiffness

$\theta_{\text {rot-int }}:$ Interior bearing stiffness

$\theta_{\text {conc }}:$ Concrete Young's modulus

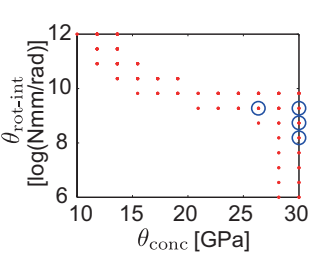

Figure 15: Pairwise comparison of parameter values that are identified using the identification framework.

\subsubsection{Prognosis and next steps}

In the next step, since the diagnostics is adequate, the 193 candidate models are used to predict displacement for LC-4 and LC-6 and to verify the diagnostics. To do this, the procedure presented in Section 2.6 is employed with $\phi_{p}=0.95$. In Eq. (8), the uncertainty term $U_{j, g_{\kappa}}$ is $U_{j, g_{4}}-U_{j, \hat{y}}$, including the measurement uncertainty, since the prognoses here is compared with measured values. The predicted ranges that are bounded by the prediction threshold includes the measured value for all locations. Figure 16 presents a prognosis example for sensor D-1-2 under LC-6 as well as the prognosis that is obtained with the initial model set (i.e. if structural identification would have not been performed). A high reduction in uncertainty

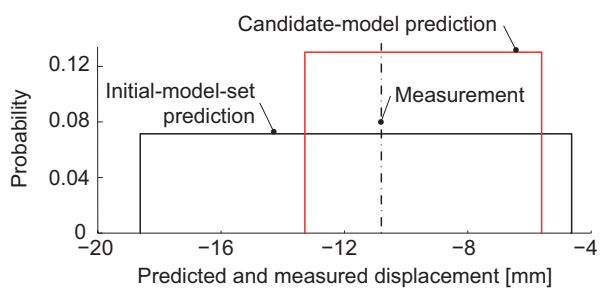

Figure 16: Comparison of prediction distribution and measured value for displacement of sensor D-1-2 under LC-6.

is revealed between the initial-model-set and candidate-model-set predictions. However, even after identification the prediction range is large (between -6 and $-13 \mathrm{~mm}$ ). This is due to the modeling uncertainties that include the non-linear behavior uncertainty which has a high influence, as presented in Table 4.

This table shows that the non-linear behavior uncertainty has $25 \%$ importance before identification, which is the highest relative importance. However, this uncertainty source cannot be identified as the parameters $\boldsymbol{\theta}_{\mathbf{4}}$ and cannot be reduced using information provided by measurements. Also, this table depicts that the parameter relative importance decreases significantly after identification showing the benefits of 
Table 4: Relative importances of parameter, modeling and measurement uncertainties for identification framework iteration 4 . The relative importance values are averaged over all displacement measurement locations.

\begin{tabular}{lllll}
\hline Uncertainty source & \multicolumn{3}{c}{ Relative importance } \\
\cline { 2 - 5 } & \multicolumn{3}{c}{ Before identification } & After identification \\
\hline Exterior bearing rotational stiffness & $10 \%$ & $5.0 \%$ & $\square$ \\
Interior bearing rotational stiffness & $23 \%$ & $6.0 \%$ & $\square$ \\
Young's modulus of concrete & $12 \%$ & $3.0 \%$ & $\square$ \\
Modeling uncertainties & $17 \%$ & $23 \%$ & \\
Measurement uncertainties & $13 \%$ & & $21 \%$ & \\
Non-linear behavior & $25 \%$ & & $42 \%$ & \\
\hline
\end{tabular}

structural identification.

Note also that using the measurements of all load cases (LC-1 to LC-6), model falsification leads to the same 4 candidate models obtained using only four load cases. This shows that the four load cases are sufficient to identify the structural behavior of this bridge.

Assuming that a prognosis of the remaining fatigue life is required, it is likely that the prognosis performance will be inadequate by extrapolating results from Figure 16. Indeed, the uncertainty associated with the predictions is too large and the source of uncertainty that is responsible for this is difficult to estimate for conditions other than those prevailing during monitoring due to the lack of knowledge of the true bearing device behavior. Two scenarios may be examined in order to guide the engineer in his decision related to the subsequent steps:

- Scenario I: Prognosis results are required. The next step should be a new monitoring task focusing on the bearing-device behavior that will lead to a new diagnostic and a more accurate prognosis.

- Scenario II: The cause of the non-linear behavior has been identified, and thus intervention is required to avoid other bridge components from being damaged by such unexpected behavior. The intervention would be the replacement of the bearing devices on the skewed side since those on the straight side have shown to have a low influence on the bridge response. Then, further monitoring is required to identify a new model class in order to carry out a revised prognosis.

In such situations, the engineer should compare the costs of both scenarios. Scenario II could be more appropriate since bearing devices are likely to be replaced as a result of scenario I. 

data.

Table 5: Summary of the iterative structural identification framework applied to US202/NJ23 Bridge.

\begin{tabular}{|c|c|c|c|}
\hline Iter. & Model class & Acquired information & Acquired knowledge \\
\hline 1 & $\begin{array}{l}\mathcal{G}_{1}=\left\{g_{1}\left(\mathbf{x}, \boldsymbol{\theta}_{1}\right)\right\} \cup\left\{\mathbf{U}_{g_{1}}\right\}^{\mathrm{a}} \\
\boldsymbol{\theta}_{1}=\left[\theta_{\text {rot }}, \theta_{\text {conc }}\right]^{\top}\end{array}$ & $\begin{array}{l}\Omega_{1}^{*}=\emptyset, \text { measurements } \\
\text { drawings }\end{array}$ & Basic knowledge \\
\hline 2 & $\begin{array}{l}\mathcal{G}_{2}=\left\{g_{2}\left(\mathbf{x}, \boldsymbol{\theta}_{2}\right)\right\} \cup\left\{\mathbf{U}_{g_{1}}+\mathbf{V}_{\text {pier }}\right\} \\
\boldsymbol{\theta}_{2}=\left[\theta_{\text {rot-ext }}, \theta_{\text {rot-int }}, \theta_{\text {conc }}\right]^{\top}\end{array}$ & $\begin{array}{l}\Omega_{2}^{*}=\emptyset, \text { deteriorations } \\
\text { (inspection) }\end{array}$ & $\begin{array}{l}\text { Pier-cap crack low impor- } \\
\text { tance, bearing non-linear } \\
\text { behavior }\end{array}$ \\
\hline 3 & $\begin{array}{l}\mathcal{G}_{3}=\left\{g_{3}\left(\mathbf{x}, \boldsymbol{\theta}_{3}\right)\right\} \cup\left\{\mathbf{U}_{g_{2}}+\sum_{r=1}^{8} \mathbf{V}_{\text {rot- } r}\right\} \\
\boldsymbol{\theta}_{3}=\left[\theta_{\text {rot-1s- } C_{1}}, \theta_{\text {rot-1s- } C_{2}}, \theta_{\text {rot-1s- } \phi}, \ldots, \theta_{\text {conc }}\right]^{\top}\end{array}$ & $\Omega_{3}^{*}=\emptyset$ & $\begin{array}{l}\text { Inadequate bilinear behav- } \\
\text { ior }\end{array}$ \\
\hline 4 & $\begin{array}{l}\mathcal{G}_{4}=\left\{g_{4}\left(\mathbf{x}, \boldsymbol{\theta}_{4}\right)\right\} \cup\left\{\mathbf{U}_{g_{3}}+\mathbf{W}_{\mathrm{nl}}\right\} \\
\boldsymbol{\theta}_{4}=\left[\theta_{\text {rot-ext }}, \theta_{\text {rot-int }}, \theta_{\text {conc }}\right]^{\top}\end{array}$ & $\Omega_{4}^{*}=\left\{\boldsymbol{\Theta}_{4}\right\}, n_{\Omega_{4}^{*}}=193$ & $\begin{array}{l}\text { Complex non-linear behav- } \\
\text { ior, inaccurate predictions }\end{array}$ \\
\hline
\end{tabular}

This example demonstrated that an iterative process is necessary for acquiring important information and knowledge to perform structural identification. Figure 17 summarizes the iterations and the steps required in this case study where arrows illustrate decisions of the engineer. Although the case study of US202/NJ23

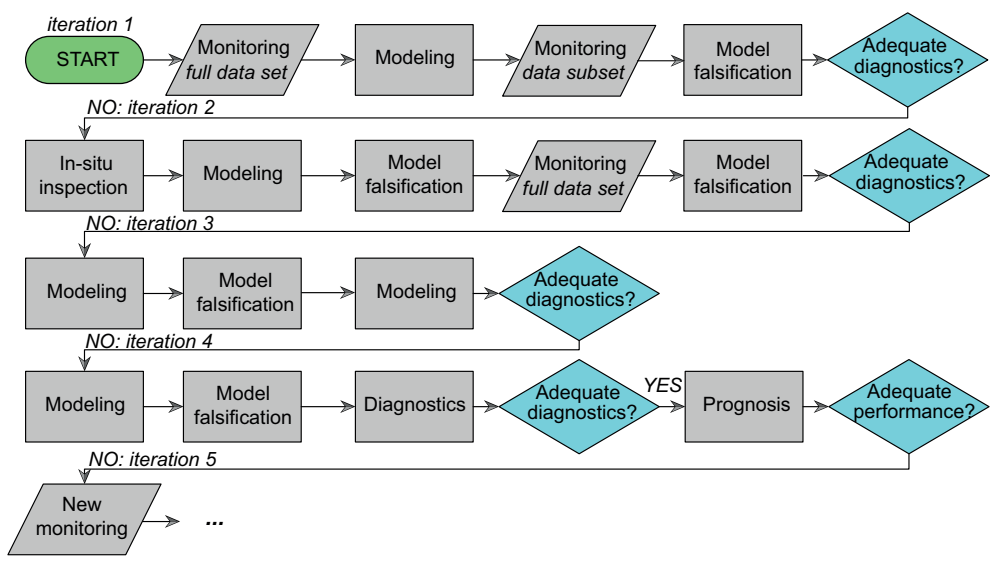

Figure 17: Iterative structural identification process for US202/NJ23 Bridge.

Bridge presents a partial structural identification process, it illustrates the process of engineers acquiring knowledge to make better decisions regarding structural health management. Indeed, starting with a naive model class originating from his basic knowledge, the iterative process increased the engineer's knowledge of the structural behavior to a state at which a decision is possible. As shown in Table 5, each iteration increases the knowledge acquired either by raw information on the structure or interpreting measurement 
In such a process, the falsification perspective and engineering heuristics play the main role, since they help engineers structure their knowledge through discarding wrong hypotheses about the structural behavior. Thus, the engineer could falsify the bilinear behavior model of the bearing and confirm the low influence of the straight-side bearing stiffness. This limits interventions to the skewed-side bearing devices. In addition, the low influence of pier-cap-cracks on bridge behavior is confirmed by identifying the 193 candidate models and thus temporarily rejecting the retrofit of this bridge component.

For complex structures, the right physics-based model is never uniquely identified. However, using the structural identification framework, engineers are supported through reasoning with discrete populations of model instances. By testing hypotheses under the form of model classes through the model-falsification task, engineers gain a better understanding of the sources of discrepancies between model predictions and measurements. Such knowledge may be then helpful for increasing accuracy related to the prognosis task.

Lack of precise prognosis originates from the fact that given available knowledge, modeling uncertainty is too high. Further investigation is required to reduce this uncertainty. While a calibrated model may always give an answer, it may not result in reliable structural identification and thus may lead to wrong predictions and unnecessary actions, particularly when extrapolating $[3,14]$.

Nevertheless, improvements are necessary in order to increase the robustness of the approach. Indeed, the non-linear behavior is identified only by LC-1 and LC-5 in addition to LC-2 and LC-3. Without the data of these load cases, a wrong model class could have been identified. In addition, the non-linear behavior uncertainty would have been difficult to estimate if the static-load test had not been carried out by step-wise increases in loading. Thus, more effort is required to determine optimal loading strategies.

\section{Conclusion}

This paper proposes a new iterative structural identification framework for the diagnosis and prognosis of existing structures. A full-scale study involving US202/NJ23 Bridge illustrates the benefits of the framework. This study leads to the following conclusions:

- This approach is able to support structural identification through combining engineering heuristics with on-site measurements and is robust to modeling systematic uncertainties.

- The iterative structural identification framework explores the compatibility of several model classes by model-class falsification.

- The study of the bridge in Wayne shows that the modeling uncertainty is dominated by complex nonlinear behavior of the bearing devices, thereby leading to the need for additional monitoring campaign for better identification. 


\section{Acknowledgements}

The authors acknowledge the Intelligent Infrastructure Alliance from Drexel University, particularly Prof. A. Emin Aktan, Prof. Franklin L. Moon and Dr. Jeff Weidner, for providing the case study and the measurement data. The authors also thank the International Bridge Study (IBS) Group that was initiated with the Center for Advanced Infrastructure and Transportation (CAIT) of Rutgers University, New Jersey Department of Transportation and Federal Highway Administration Long-Term Bridge Performance Program support and located in Rutgers. The authors also thank Dr. James-A. Goulet for support and fruitful discussions. This work was funded by the Swiss National Science Foundation under Contract no. 200020-155972.

\section{References}

[1] S. Atamturktur, Z. Liu, H. Cogan, S.and Juang, Calibration of imprecise and inaccurate numerical models considering fidelity and robustness: a multi-objective optimization-based approach, Structural and Multidisciplinary Optimization (2014) 1-13.

[2] J. Beck, Bayesian system identification based on probability logic, Structural Control and Health Monitoring 17 (7) (2010) 825-847.

[3] J.-A. Goulet, I. Smith, Structural identification with systematic errors and unknown uncertainty dependencies, Computers \& Structures 128 (2013) 251-258.

[4] F. Çatbaş, T. Kijewski-Correa, A. Aktan, Structural identification of constructed systems, Reston (VI): American Society of Civil Engineers .

[5] J. Beck, L. Katafygiotis, Updating models and their uncertainties. I: Bayesian statistical framework, Journal of Engineering Mechanics 124 (4) (1998) 455-461.

[6] B. Goller, G. Schuëller, Investigation of model uncertainties in Bayesian structural model updating, Journal of Sound and Vibration 330 (25) (2011) 6122-6136.

[7] H. Lam, H. Peng, S. Au, Development of a practical algorithm for Bayesian model updating of a coupled slab system utilizing field test data, Engineering Structures 79 (2014) $182-194$.

[8] J. Beck, K.-V. Yuen, Model selection using response measurements: Bayesian probabilistic approach, Journal of Engineering Mechanics 130 (2) (2004) 192-203.

[9] K. Worden, J. J. Hensman, Parameter estimation and model selection for a class of hysteretic systems using Bayesian inference, Mechanical Systems and Signal Processing 32 (2012) 153-169.

[10] C. Argyris, P. Tsopelas, C. Papadimitriou, Bayesian Uncertainty Quantification in Seismically Isolated Structures Equipped with Nonlinear Hysteretic Devices, in: J. Rodellar, A. Güemes, F. Pozo (Eds.), Proceedings of the 6th World Conference on Structural Control and Monitoring (WCSCM), Barcelona, Spain, 2478-2488, 2014.

[11] N. Dubbs, F. Moon, Comparison and Implementation of Multiple Model Structural Identification Methods, Journal of Structural Engineering in press (2015) 04015042. 
[12] B. Goller, J. Beck, G. Schuëller, Evidence-Based Identification of Weighting Factors in Bayesian Model Updating Using Modal Data, Journal of Engineering Mechanics 138 (2012) 430-440.

[13] D. Straub, I. Papaioannou, Bayesian updating with structural reliability methods, Journal of Engineering Mechanics 141 (3) (2014) 04014134.

[14] R. Pasquier, I. F. C. Smith, Robust system identification and model predictions in the presence of systematic uncertainty, Advanced Engineering Informatics (2015) in press.

[15] J. Ching, Y.-C. Chen, Transitional Markov chain Monte Carlo method for Bayesian model updating, model class selection, and model averaging, Journal of Engineering Mechanics 133 (7) (2007) 816-832.

[16] M. Muto, J. Beck, Bayesian updating and model class selection for hysteretic structural models using stochastic simulation, Journal of Vibration and Control 14 (1-2) (2008) 7-34.

[17] K.-V. Yuen, Bayesian methods for structural dynamics and civil engineering, Wiley, 2010.

[18] D. Mackay, Information theory, inference and learning algorithms, Cambridge University Press, 2003.

[19] K. Van Buren, T. Hall, L. Gonzales, F. Hemez, S. Anton, A case study to quantify prediction bounds caused by model-form uncertainty of a portal frame, Mechanical Systems and Signal Processing 50-51 (2015) 11-26.

[20] E. Simoen, G. D. Roeck, G. Lombaert, Dealing with uncertainty in model updating for damage assessment: A review, Mechanical Systems and Signal Processing 56-57 (2015) 123-149.

[21] P. Reichert, M. Omlin, On the usefulness of overparameterized ecological models, Ecological Modelling 95 (2) (1997) $289-299$.

[22] C. Chatfield, Model Uncertainty, Data Mining and Statistical Inference, Journal of the Royal Statistical Society. Series A (Statistics in Society) 158 (3) (1995) 419-466.

[23] E. Simoen, C. Papadimitriou, G. Lombaert, On prediction error correlation in Bayesian model updating, Journal of Sound and Vibration 332 (18) (2013) 4136-4152.

[24] H. Choi, K. Beven, Multi-period and multi-criteria model conditioning to reduce prediction uncertainty in an application of TOPMODEL within the GLUE framework, Journal of Hydrology 332 (34) (2007) 316-336.

[25] K. Beven, A. Binley, The future of distributed models: Model calibration and uncertainty prediction, Hydrological Processes 6 (3) (1992) 279-298.

[26] M. Beck, Water quality modeling: a review of the analysis of uncertainty, Water Resources Research 23 (8) (1987) 13931442 .

[27] N. Cherpeau, G. Caumon, J. Caers, B. Lévy, Method for Stochastic Inverse Modeling of Fault Geometry and Connectivity Using Flow Data, Mathematical Geosciences (2012) 1-22.

[28] M. Yarnold, F. Moon, A. Aktan, Temperature-Based Structural Identification of Long-Span Bridges, Journal of Structural Engineering in press (2015) 04015027.

[29] M. Fontan, D. Breysse, F. Bos, A hierarchy of sources of errors influencing the quality of identification of unknown parameters using a meta-heuristic algorithm, Computers \& Structures 139 (2014) 1-17. 
[30] J. Baroth, Y. Malecot, Probabilistic analysis of the inverse analysis of an excavation problem, Computers and Geotechnics 37 (3) (2010) 391-398.

[31] H. Schlune, M. Plos, K. Gylltoft, Improved bridge evaluation through finite element model updating using static and dynamic measurements, Engineering Structures 31 (7) (2009) 1477-1485.

[32] F. Moon, A. Aktan, Impacts of epistemic (bias) uncertainty on structural identification of constructed (civil) systems, Shock and Vibration Digest 38 (5) (2006) 399-422.

[33] A. Jakeman, R. Letcher, J. Norton, Ten iterative steps in development and evaluation of environmental models, Environmental Modelling \& Software 21 (5) (2006) 602-614.

[34] A. Aamodt, A case-based answer to some problems of knowledge-based systems, in: Scandinavian Conference on Artificial Intelligence-93: Proceedings of the Fourth Scandinavian Conference on Artificial Intelligence Electrum, Stockholm, Sweden, May 4-7, 1993, vol. 18, IOS Press, 168, 1993.

[35] K. Popper, The logic of scientific discovery, 3rd ed. New York, Routledge, 2002.

[36] A. Tarantola, Popper, Bayes and the inverse problem, Nature Physics 2 (8) (2006) 492-494.

[37] K. Beven, A manifesto for the equifinality thesis, Journal of Hydrology 320 (1-2) (2006) 18-36.

[38] R. Pasquier, I. Smith, Sources and forms of modelling uncertainties for structural identification, in: Proceedings of 7 th International Conference on Structural Health Monitoring of Intelligent Infrastructure (SHMII), Torino, IT, 2015.

[39] S. Atamturktur, F. Hemez, J. Laman, Uncertainty quantification in model verification and validation as applied to large scale historic masonry monuments, Engineering Structures 43 (2012) 221-234.

[40] K. Van Buren, M. Mollineaux, F. Hemez, S. Atamturktur, Simulating the dynamics of wind turbine blades: part II, model validation and uncertainty quantification, Wind Energy 16 (5) (2013) 741-758.

[41] J.-A. Goulet, C. Michel, I. Smith, Hybrid probabilities and error-domain structural identification using ambient vibration monitoring, Mechanical Systems and Signal Processing 37 (1-2) (2013) 199-212.

[42] J.-A. Goulet, M. Texier, C. Michel, I. Smith, L. Chouinard, Quantifying the Effects of Modeling Simplifications for Structural Identification of Bridges, Journal of Bridge Engineering 19 (1) (2014) 59-71.

[43] R. Pasquier, J.-A. Goulet, C. Acevedo, I. Smith, Improving Fatigue Evaluations of Structures Using In-service Behavior Measurement Data, Journal of Bridge Engineering 19 (11) (2014) 04014045.

[44] J.-A. Goulet, I. Smith, Performance-Driven Measurement System Design for Structural Identification, Journal of Computing in Civil Engineering 27 (4) (2013) 427-436.

[45] R. Pasquier, J.-A. Goulet, I. Smith, Model-Based Data Interpretation and Diagnosis Robustness, in: Deodatis, Ellingwood, Frangopol (Eds.), Safety, Reliability, Risk and Life-Cycle Performance of Structures \& Infrastructures. Proceedings of 11th International Conference on Structural Safety \& Reliability (ICOSSAR), New York, USA, 2497-2504, 2014.

[46] M. Papadopoulou, B. Raphael, I. Smith, C. Sekhar, Hierarchical Sensor Placement Using Joint Entropy and the Effect of Modeling Error, Entropy 16 (9) (2014) 5078-5101. 